\title{
Distributed fiber optic radiation sensors
}

\author{
Aleksander Wosniok and Katerina Krebber \\ Bundesanstalt für Materialforschung und -prüfung (BAM), Berlin, 12205, Germany \\ Correspondence: Aleksander Wosniok (aleksander.wosniok@bam.de)
}

Published: 10 November 2021

\begin{abstract}
The international research efforts focused on the development of radiation sensors based on optic fibers have their origins in the 1970s (Evans et al., 1978). Generally, the lightweight fiber optic sensors are immune to electromagnetic field interference and high voltages making them deployable in harsh environments at hard to reach areas where conventional sensors usually will not work at all. A further advantage of such radiation sensors is the possibility of remote and real-time monitoring (Huston et al., 2001). In this work, we present our results achieved in several research activities for development of fiber optic dosimeters. The findings show that both the measurement of the radiation-induced attenuation (RIA) along the entire sensing fiber and the accompanying change in the refractive index of the fiber core can be used for distributed radiation monitoring in the kGy and MGy range, respectively. Depending on the fiber type and material the RIA shows varying response to dose rates, environmental temperatures and the wavelength of the laser source used. Thereby, an operation with visible laser light provides most favorable performance in terms of high radiation sensitivity. Operating at these wavelengths, RIA monitoring could yield high-sensitivity dose measurement with sub-gray resolution and accuracy (Stajanca and Krebber, 2017b); however, conventional optical time-domain reflectometry (OTDR) systems for RIA measurements operating in the visible range suffer from low-spatial resolution, long measurement times and poor signal-to-noise (SNR) ratio. The limitations of the OTDR performance can be overcome by the incoherent optical frequency domain reflectometry (I-OFDR) developed by the Federal Institute of Materials Research and Testing (BAM, Liehr et al., 2009) with potential for dynamic real-time measurement. Over the years, several highly radiation sensitive fibers, such as perfluorinated polymer optical fibers (PF-POF, Stajanca and Krebber, 2017a), phosphorous-doped silica optical fibers (SOF, Paul et al., 2009), aluminium-doped SOF (Faustov et al., 2013) and erbium-doped SOF (Wosniok et al., 2016) have been identified and are commercially available. As mentioned before, the radiation-induced RIA increase is associated with an increase in the refractive index leading also to material compaction in the fiber core. The latter two effects can be used for measuring radiation distribution based on Brillouin scattering in the range of high radiation doses of several MGy (Phéron et al., 2012; Wosniok et al., 2016). When using fiber optic sensors for radiation monitoring, the existing post-irradiation annealing behavior of the optical fiber sensors must also be considered.
\end{abstract}

Kurzfassung. Die internationalen Forschungsanstrengungen zur Entwicklung von Strahlungssensoren auf Basis optischer Fasern haben ihren Ursprung in den 1970ern (Evans et al., 1978). Im Allgemeinen werden die leichten faseroptischen Sensoren durch elektromagnetische Feldstörungen und hohe Spannungen nicht beeinträchtigt, wodurch sie in rauen Umgebungen an schwer zugänglichen Stellen eingesetzt werden können, an denen herkömmliche Sensoren in der Regel überhaupt nicht funktionieren. Ein weiterer Vorteil solcher Strahlungssensoren ist die Möglichkeit der Fern- und Echtzeitüberwachung (Huston et al., 2001). In dieser Arbeit stellen wir unsere aus mehreren Forschungsarbeiten gewonnenen Ergebnisse zur Entwicklung faseroptischer Dosimeter vor. Sie zeigen, dass sowohl die Messung der strahlungsinduzierten Dämpfung (RIA: „radiation-induced attenuation") entlang der gesamten Messfaser als auch die damit einhergehende Änderung des Brechungsindex des Faserkerns für die Strahlungsüberwachung im kGy- bzw. MGy-Bereich genutzt werden können. Abhängig von Fasertyp und Material reagiert die RIA unterschiedlich auf Dosisleistungen, Umgebungstemperaturen und die Wellenlänge der verwendeten Laserquelle. Dabei bietet ein Betrieb mit sichtbarem Laserlicht die günstigste Leistung im Hinblick auf eine hohe Strahlungsempfindlichkeit. Bei diesen Wellenlängen könnte die RIAÜberwachung eine hochempfindliche Dosisbestimmung mit Auflösung und Genauigkeit im Sub-Gy-Bereich 
ergeben (Stajanca und Krebber, 2017b). Herkömmliche optische Zeitbereichsreflektometer (OTDR systems [OTDR: „time-domain reflectometry“]) für RIA-Messungen, die im sichtbaren Bereich arbeiten, haben jedoch nur eine geringe räumliche Auflösung, lange Messzeiten und ein schlechtes Signal-Rausch-Verhältnis (SNR: „signal-to-noise ratio“). Diese Einschränkungen der OTDR-Leistung können durch die von der BAM (Bundesanstalt für Materialforschung und -prüfung, Liehr et al., 2009) entwickelte inkohärente optische Frequenzbereichsreflektometrie (I-OFDR: ,incoherent optical frequency domain reflectometry“) mit Potenzial für dynamische Echtzeitmessungen überwunden werden. Im Laufe der Jahre wurden mehrere hochstrahlungsempfindliche Fasern, wie perfluorierte optische Polymerfasern (PF-POF; Stajanca und Krebber, 2017a), mit Phosphor versetzte optische Quarzglasfasern (SOF; Paul et al., 2009), mit Aluminium angereicherte SOF (Faustov et al., 2013) und SOF mit Erbiumzusatz (Wosniok et al., 2016), identifiziert und sind im Handel erhältlich. Wie bereits erwähnt, ist die strahlungsinduzierte RIA-Zunahme mit einer Erhöhung des Brechungsindex assoziiert, was auch zu einer Materialverdichtung im Faserkern führt. Die beiden letztgenannten Effekte können zur Messung der Strahlungsverteilung auf Basis der Brillouin-Streuung im Bereich hoher Strahlendosen von mehreren MGy genutzt werden (Phéron et al., 2012; Wosniok et al., 2016). Beim Einsatz von faseroptischen Sensoren zur Strahlungsüberwachung muss auch deren Nachglühverhalten nach Bestrahlung berücksichtigt werden.

\section{References}

Evans, B. D., Sigel, G. H., Langworthy, J. B., Faraday, B. J., and Chang, T. C.: The fiber optic dosimeter on the navigational technology satellite, IEEE T. Nucl. Sci., 25, 1619-1624, https://doi.org/10.1109/TNS.1978.4329582, 1978.

Faustov, A. V., Gusarov,, A., Wuilpart, M., Fotiadi, A. A., Liokumovich, L. B., Zolotovskiy, I. O., Thomashuk, A. L., de Schoutheete, T., and Megret, P.: Comparison of gammaradiation induced attenuation in Al-doped, P-doped and Gedoped fibres for dosimetry, IEEE T. Nucl. Sci., 60, 2511-2517, https://doi.org/10.1109/TNS.2013.2273273, 2013.

Huston A. L., Justus, B. L., Falkstein, P. L., Miller, R. W., Ning, H., and Altemus, R.: Remote optical fiber dosimetry, Nucl. Instrum. Meth. B, 184, 55-67, https://doi.org/10.1016/S0168583X(01)00713-3, 2001.

Liehr, S., Nöther, N., and Krebber, K.: Incoherent optical frequency domain reflectometry and distributed strain detection in polymer optical fibers, Meas. Sci. Technol., 21, 017001, https://doi.org/10.1088/0957-0233/21/1/017001, 2009.
Paul, M. C., Bohra, D., Dhar, A., Sen, R., Bhatnagar, P. K., and Dasgupta, K.: Radiation response behavior of high phosphorous doped step-index multimode optical fibers under low dose gamma irradiation, J. Non-Cryst. Solids, 355, 1496-1507, https://doi.org/10.1016/j.jnoncrysol.2009.05.017, 2009.

Phéron, X., Girard, S., Boukenter, A., Brichard, B., DelepineLesoille, S., Bertrand, J., and Ouerdane, Y.: High $\gamma$-ray dose radiation effects on the performances of Brillouin scattering based optical fiber sensors, Opt. Express, 20, 26978-26985, https://doi.org/10.1364/OE.20.026978, 2012.

Stajanca, P. and Krebber, K.: Radiation-induced attenuation of perfluorinated polymer optical fibers for radiation monitoring, Sensors, 17, 1959, https://doi.org/10.3390/s17091959, 2017a.

Stajanca, P. and Krebber, K.: Polymer optical fibers as radiation sensors?, in: Proc. of POF2017, 13-15 September 2017, Aveiro, Portugal, 2017b.

Wosniok, A., Sporea, D., Neguţ, D., and Krebber, K.: Gamma radiation influence on silica optical fibers measured by optical backscatter reflectometry and Brillouin sensing technique, Proc. SPIE, 9916, 99162J, https://doi.org/10.1117/12.2236678, 2016. 